\title{
Clinical course of severe esophagitis following bevacizumab therapy
}

\author{
Jun-Ho Choi and Kwangwoo Nam
}

Department of Internal Medicine, Dankook University Hospital, Dankook University College of Medicine, Cheonan, Korea
Received : March 11, 2021

Revised : March 22, 2021

Accepted: March 23, 2021

\section{Correspondence to}

Kwangwoo Nam, M.D.

Tel: +82-41-550-3092

Fax: +82-41-550-7050

E-mail: nambag1108@gmail.com https://orcid.org/0000-00033720-9820
A 57-year-old woman presented with worsening chest pain, dysphagia, and vomiting. She denied having prior episodes of heartburn or acid regurgitation. Nine months prior, she had been diagnosed with tubal cancer with peritoneal metastasis. The patient was treated with neoadjuvant chemotherapy using paclitaxel and carboplatin, followed by radical cytoreductive surgery, and subsequent adjuvant chemotherapy using paclitaxel and cisplatin was completed. However, 5 months after surgery, peritoneal recurrence was detected. Thus, palliative chemotherapy with bi-weekly bevacizumab and liposomal doxorubicin was initiated, but after administration of bevacizumab, the patient suffered from severe upper
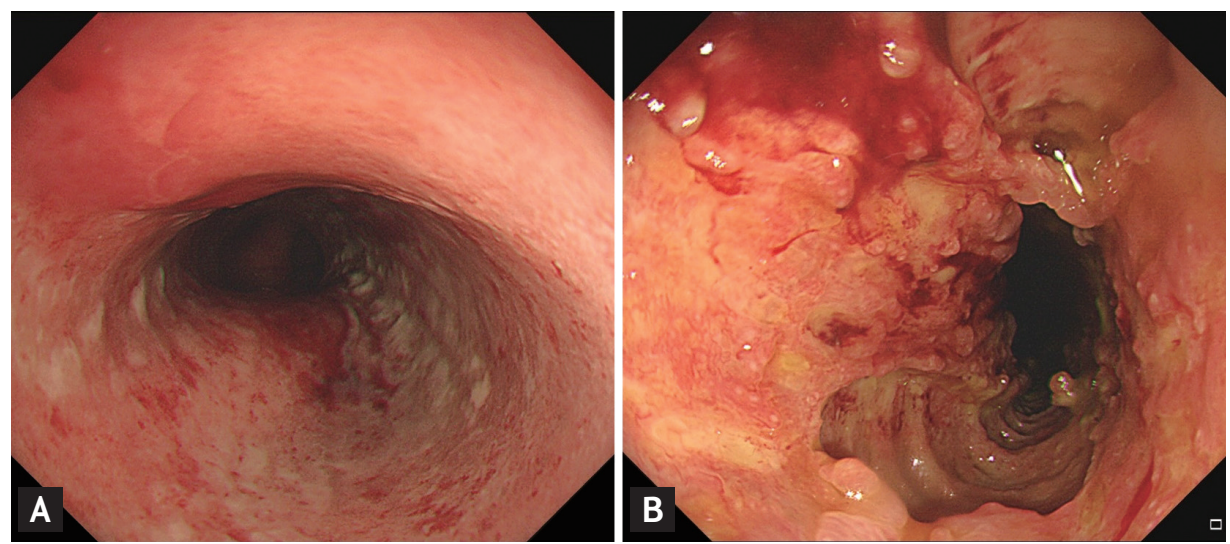

Figure 1. (A) Esophagogastroduodenoscopy images obtained at the first cycle of palliative chemotherapy. Linear erosive esophagitis is observed in the middle esophagus. (B) Esophagogastroduodenoscopy images obtained at the third cycle of palliative chemotherapy. Multiple severe ulcers with impending perforation are observed in the middle esophagus. 


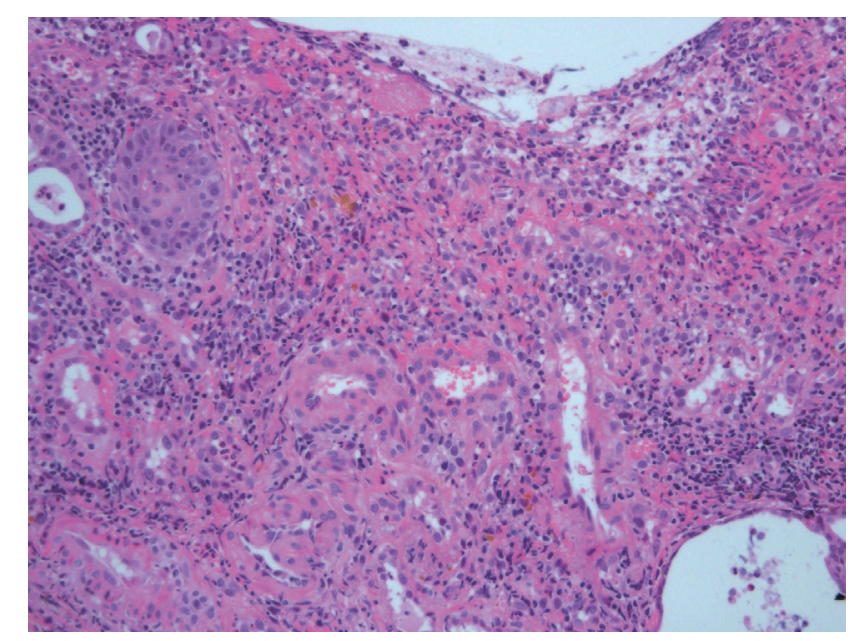

Figure 2. Histopathologic findings of endoscopic forceps biopsy. Superficial surface erosion displays fibrinopurulent exudates and stroma displays mixed inflammatory cells and increased vasculature. There was no sign of cytomegalovirus infeciton or malignant cell $(H \& E, \times 200)$.

cell (Fig. 2). Serum cytomegalovirus antibody was also negative. These results suggested bevacizumab-induced severe esophageal injury. Therefore, bevacizumab therapy was discontinued and proton pump inhibitor was maintained, resulting in gradual improvement in upper gastrointestinal symptoms. Informed consent was obtained from the patient.

Although bevacizumab is generally well tolerated, several serious adverse events including gastrointestinal perforation had been reported. Bevacizumab is an anti-vascular endothelial growth factor monoclonal antibody; thus, vascular injury and ischemia of the gastrointestinal tract is thought to contribute to these complications. When patients taking bevacizumab develop severe upper gastrointestinal symptoms, prompt endoscopic evaluation should be considered to enable early diagnosis and proper treatment.

\section{Conflict of interest}

No potential conflict of interest relevant to this article was reported.

\section{Acknowledgments}

The present research was conducted by the research fund of Dankook University in 2019. 